\title{
Multi-criteria decision making method of minimal suitable values
}

\author{
MALiŠA ŽižOVIĆ, NADA DAMLJANOviĆ, \\ RAle NikOlić AND MOMČIlo VujiČIĆ
}

\begin{abstract}
This paper presents a conceptual and mathematical model of a multi-criteria decision making process in which the information about decision maker's preferences is expressed in the form of minimal suitable values.
\end{abstract}

\section{INTRODUCTION}

The multi-criteria decision making can be viewed as a process of identifying the most preferred alternative choice or a process of ranking the alternatives based on a set of criteria, which usually have a different significance to the decision. During the previous decades, multi-criteria decision analysis was one of the fastest growing areas and many multi-criteria decision methods and techniques were proposed and elaborated $[1,2,3,9,10$, 11, 13, 18, 19]. Each method has its own characteristics, but they all include following steps in processing: determining the relevant criteria and alternatives, associating weights to criteria and performance values to the alternatives on these criteria, and finally performing appropriate aggregation procedure. Selected alternatives must be real, practical, available and comparable. Selected criteria must be independent of each other, represented in same scale, measurable and related with the alternatives. The choice of the aggregation function to be used in a model of multi-criteria decision making problem directly depend on properties dictated by the framework in which the aggregation is performed. The weighted sum is the earliest and probably the most widely used aggregation function, but other aggregation functions can also be applied $[5,7,8]$.

The diversity of multi-criteria decision making methods emerges from different design problems to be solved. A large number of methods have been well investigated from the mathematical point of view and applied in a wide variety of fields [6]. In combination with the use of modern computers many of these methods have had an extensive software support. However, the problem of choosing an appropriate method in a particular situation

Key words and phrases. Multicriteria analysis, alternative, criterion, weight. 
still exists which encourages researchers to develop new techniques. For an overview of the available methods for solving multi-criteria decision problems we refer to Figueira et al. [4], Hwang and Yoon [7], Radojičić and Žižović [12], Triantaphyllou [15] and Zeleny [17].

\section{Preliminaries}

Let $A=\left\{a_{1}, a_{2}, \ldots, a_{m}\right\}$ be the set of alternatives that are to be assessed by the set of criteria $C=\left\{c_{1}, c_{2}, \ldots, c_{n}\right\}$. For every $i=1,2, \ldots, m$, the alternative $a_{i}$ can be represented as $n$-tuple $a_{i}=\left(a_{i 1}, a_{i 2}, \ldots, a_{i n}\right)$, or equivalently $a_{i}=\left(a_{i k}\right)_{k=1}^{n}$, where each coordinate $a_{i j}$, for $j=1,2, \ldots, n$, is a nonnegative real number which can be interpreted as the degree to which the alternative $a_{i}$ satisfies the criterion $c_{j}$. In this way, the multi-criteria decision problem can be presented by a decision matrix given by Table 1 .

TABLE 1. Decision matrix.

\begin{tabular}{|c|c|c|l|c|}
\hline & $C_{1}$ & $C_{2}$ & $\cdots$ & $C_{n}$ \\
\hline$A_{1}$ & $a_{11}$ & $a_{12}$ & $\cdots$ & $a_{1 n}$ \\
\hline$A_{2}$ & $a_{21}$ & $a_{22}$ & $\cdots$ & $a_{2 n}$ \\
\hline$\vdots$ & $\vdots$ & $\vdots$ & $\ddots$ & $\vdots$ \\
\hline$A_{m}$ & $a_{m 1}$ & $a_{m 2}$ & $\cdots$ & $a_{m n}$ \\
\hline
\end{tabular}

The natural assumption is that all members of the set $A$ are mutually incomparable under standard order relation $\leq_{A^{n}}$ on $A^{n}$, i.e. when an alternative is better according to one criterion, the other is better according to the other criterion.

As it is usual in the multi criteria decision making analysis, we will assume that criteria can have different importance to the decision and that the decision makers are aware of the degree of importance of each criterion. In other words, we will assume that each criterion $c_{k}$, for $k=1,2, \ldots, n$, is associated with the weight $w_{k} \in(0,1]$ that represents the degree of importance of that criterion, and moreover, we will assume that the weights are given in a normalized form, i.e.

$$
\sum_{k=1}^{n} w_{k}=1 .
$$

Notice that the criteria weights can be settled either by the user or the preference information is collected through a pair-wise comparison of the previously selected alternatives (for example, see AHP-method [14]). For an overview on various weighting methods we referee to [16]. 
For a given set of alternatives $A=\left\{a_{1}, a_{2}, \ldots, a_{n}\right\}$, the upper set $\bar{A}$ and the lower set $\underline{A}$ are defined by

$$
\begin{aligned}
& \bar{A}=\left\{\left(\max _{i \in J} a_{i k}\right)_{k=1}^{n} \mid J \in \mathcal{P}(\{1,2, \ldots, m\}) \backslash\{\emptyset\}\right\}, \\
& \underline{A}=\left\{\left(\min _{i \in J} a_{i k}\right)_{k=1}^{n} \mid J \in \mathcal{P}(\{1,2, \ldots, m\}) \backslash\{\emptyset\}\right\} .
\end{aligned}
$$

The max operation on $\bar{A}$ is defined pointwise, i.e.,

$$
\max \{a, b\}=\left(\max \left\{a_{k}, b_{k}\right\}\right)_{k=1}^{n}, \quad \text { for every } a, b \in \bar{A},
$$

so, the natural order relation $\leq_{\bar{A}}$ on $\bar{A}$ is given by

$$
a \leq_{\bar{A}} b \quad \Longleftrightarrow \quad \max \{a, b\}=b, \quad \text { for every } a, b \in \bar{A} .
$$

Therefore, $\left(\bar{A}, \leq_{\bar{A}}\right)$ is an upper semilattice with the greatest element

$$
\top=\max \{a \mid a \in \bar{A}\},
$$

which will be called the best hypothetical alternative. Clearly, holds

$$
\top=\left(\top_{k}\right)_{k=1}^{n}=\left(\max \left\{a_{i k} \mid i=1,2, \cdots, m\right\}\right)_{k=1}^{n} .
$$

On the other hand, the pointwise defined min operation on $\underline{A}$,

$$
\min \{a, b\}=\left(\min \left\{a_{k}, b_{k}\right\}\right)_{k=1}^{n}, \quad \text { for every } a, b \in \underline{A},
$$

induce the natural order relation $\leq_{\underline{A}}$ on $\underline{A}$ defined by

$$
a \leq_{\underline{A}} b \quad \Longleftrightarrow \quad \min \{a, b\}=a, \quad \text { for every } a, b \in \underline{A},
$$

so, $\left(\underline{A}, \leq_{A}\right)$ is an lower semilattice with the least element

$$
\perp=\min \{a \mid a \in \underline{A}\},
$$

and holds

$$
\perp=\left(\perp_{k}\right)_{k=1}^{n}=\left(\min \left\{a_{i k} \mid i=1,2, \cdots, m\right\}\right)_{k=1}^{n} .
$$

Further, we will consider the set $L=\bar{A} \cup \underline{A}$. Clearly, $A=\bar{A} \cap \underline{A}$ and therefore $A \subseteq L$. Let $\leq_{L}$ be a partial order relation on $L$ that preserves the orders on $\bar{A}$ and $\underline{A}$, i.e., for every $a, b \in L$

$a \leq_{L} b \Longleftrightarrow\left\{\begin{array}{l}a, b \in \bar{A} \text { and } a \leq_{\bar{A}} b ; \\ a, b \in \underline{A} \text { and } a \leq_{\underline{A}} b ; \\ a \in \underline{A}, b \in \bar{A} \text { and for some } a_{i} \in A, a \leq_{\underline{A}} a_{i} \text { and } a_{i} \leq_{\bar{A}} b .\end{array}\right.$

Then, let $\left(L, \leq_{L}\right)$ is a partially ordered set which can be viewed as the sum of the lower semilattice $\left(\underline{A}, \leq_{A}\right)$ and the upper semilattice $\left(\bar{A}, \leq_{\bar{A}}\right)$. The following theorem holds.

Theorem 1. $\left(L, \leq_{L}\right)$ is a lattice with the greatest element $T$ and the least element $\perp$. 
Proof. We will prove that any two-element subset of $L$ has supremum and infimum in $L$.

Let $a, b \in \bar{A}$. Then $a=\max _{i \in I}\left\{a_{i}\right\}$ and $b=\max _{j \in J}\left\{a_{j}\right\}$, for some index sets $I, J \subseteq\{1,2, \ldots, m\}$, and alternatives $a_{i} \in A, i \in I$ and $a_{j} \in A, j \in J$. By the construction of $\bar{A}$, we have that $\max \{a, b\}=\max _{l \in I \cup J}\left\{a_{l}\right\} \in \bar{A}$ is the least upper bound of $\{a, b\}$ in $L$. Let $K=I \cap J$. If $K=\emptyset$, then $c=\min _{l \in I \cup J}\left\{a_{l}\right\}$ is the greatest lower bound of $\{a, b\}$ in $L$, and in case that $K \neq \emptyset$ we have that the element $c=\max _{k \in K}\left\{a_{k}\right\}$ is the greatest lower bound of $\{a, b\}$ in $L$. Thus, supremum and infimum exist for any two-element subset of $\bar{A}$.

In a similar way we can prove that supremum and infimum exist for any two-element subset of $\underline{A}$.

Now, let $a \in \bar{A}$ and $b \in \underline{A}$. Then $a=\max _{i \in I}\left\{a_{i}\right\}$ and $b=\min _{j \in J}\left\{a_{j}\right\}$, and we have that $\max \{a, b\}=\max _{l \in I \cup J}\left\{a_{l}\right\}$ and $\min \{a, b\}=\max _{l \in I \cup J}\left\{a_{l}\right\}$.

Thus, $\left(L, \leq_{L}\right)$ is a lattice. Clearly, $\mathrm{T}=\max \bar{A}$ and $\perp=\min \underline{A}$, respectively, are the greatest and the least elements of this lattice.

For each criterion $c_{k}, k \in\{1,2, \ldots, n\}$, we will consider a function that measures the importance of the difference between two alternatives $a, b \in L$. These functions, are determined by certain common agreement between the decision maker (person that makes decisions) and the analyst (person that performs calculation). In other words, for each criterion $c_{k}, k=1,2, \ldots, n$, we will consider the specific functions $\mathcal{Z}_{k}: L \times L \rightarrow[0,+\infty)$, which joins a nonnegative real number $\mathcal{Z}_{k}(a, b)$ to each pair of alternatives $a, b \in L$. These functions were introduced by Žižović et al. [18] with the goal to implement both, the distance between alternatives and the criteria performance of alternatives into the overall aggregation values.

\section{NEW METHOD}

In this section, we will present a new multi-criteria decision making method which includes the minimal suitable values into the calculation of the alternative preferences. In this way, the decision maker includes his own expectations and express his personal preferences, and therefore, provides better insight into advantages and disadvantages of particular alternatives.

Let a list of minimal suitable values

$$
P_{q}=\left(P_{q k}\right)_{k=1}^{n}, \quad q=1,2, \ldots, s,
$$

be settled by the decision maker. We will assume that the decision maker determines mutually incomparable minimal suitable values.

For each $P_{q}, q=1,2, \ldots, s$, let

$$
F_{q}=\left\{a \in L \mid P_{q} \leq_{A^{n}} a\right\} .
$$

Theorem 2. For $q=1,2, \ldots, s$, if $F_{q}$ is a nonempty subset of $L$, then $F_{q}$ is a filter of $L$. 
Proof. For some $q=1,2, \ldots, s$, let $F_{q}$ be a nonempty set, and let $a, b \in F_{q}$. Then from $P_{q} \leq_{A^{n}} a$ and $P_{q} \leq_{A^{n}} b$ we obtain $P_{q} \leq_{A^{n}} \min _{A^{n}}\{a, b\}$. Similar as in proof of Theorem 1, we can obtain $P_{q} \leq A^{n} \min _{L}\{a, b\}$. Therefore, $\min _{L}\{a, b\} \in F_{q}$.

Further, let $a \in L, b \in F_{q}$ and $b \leq_{L} a$. Since $b \leq_{L} a$ implies $b \leq_{A^{n}} a$ and $b \in F_{q}$ implies $P_{q} \leq A^{n} b$, we have $P_{q} \leq_{A^{n}} a$, and therefore $a \in F_{q}$. Thus, $F_{q}$ is a filter of $L$.

Corollary 1. A nonempty $F_{q}$ is a principal filter of $L$ generated by

$$
f_{q}=\left(f_{q k}\right)_{k=1}^{n}=\left(\min _{b \in F_{q}} b_{k}\right)_{k=1}^{n},
$$

where $b=\left(b_{k}\right)_{k=1}^{n}$.

Proof. It is a matter of routine to verify that $f_{q} \in F_{q} \subseteq L$, so $F_{q}$ is a principal filter of $L$ generated by $f_{q}$.

For every $k=1,2, \ldots, n$ and $q=1,2, \ldots, s$, let a value $\nu_{k}\left(P_{q}, f_{q}\right) \in[0,1]$ be defined by

$$
\nu_{k}\left(P_{q}, f_{q}\right)=\left\{\begin{array}{cl}
\frac{f_{q k}-P_{q k}}{T_{k}-P_{q k}}, & \text { if } P_{q} \leq_{A^{n}} a_{i}, \text { for some } i=1,2, \ldots, m, \\
0, & \text { otherwise. }
\end{array}\right.
$$

In this way, each $P_{q}, q=1,2, \ldots, s$, is associated with the $n$-tuple

$$
\left(\nu_{1}\left(P_{q}, f_{q}\right), \nu_{2}\left(P_{q}, f_{q}\right), \ldots, \nu_{n}\left(P_{q}, f_{q}\right)\right) .
$$

Further, let $x_{1}, x_{2} \in L$ be two alternatives such that $x_{2}$ covers $x_{1}$, meaning that $x_{2}>_{L} x_{1}$ and $x_{2}>_{L} x>_{L} x_{1}$ is not satisfied by any $x \in L$. Then for every criterion $c_{k}, k=1,2, \ldots, n$, we define the preference of the alternative $x_{2}$ over the alternative $x_{1}$ with respect to the criterion $c_{k}$ by

$$
\delta_{k}\left(x_{1}, x_{2}\right)=\mathcal{Z}_{k}\left(x_{1}, x_{2}\right) \cdot \frac{x_{2 k}-x_{1 k}}{T_{k}-\perp_{k}},
$$

and we will use the following notation

$$
x_{1} \stackrel{\delta_{k}\left(x_{1}, x_{2}\right)}{\longmapsto} x_{2} .
$$

Let $a, b \in L$ and let $\pi$ be a path in $L$ from $b$ to $a$, i.e., let there exist $x_{1}, x_{2}, \ldots, x_{j} \in L$ such that

$$
\pi: \quad b=x_{1} \stackrel{\delta_{k}\left(x_{1}, x_{2}\right)}{\longmapsto} x_{2} \stackrel{\delta_{k}\left(x_{2}, x_{3}\right)}{\longmapsto} \ldots \stackrel{\delta_{k}\left(x_{j-1}, x_{j}\right)}{\longmapsto} x_{j}=a .
$$

This will be shortly denoted by

$$
\pi: b \longrightarrow a .
$$

Then the running preference of the alternative $a$ over the alternative $b$ with respect to the criterion $c_{k}$ trough the path $\pi$ is defined as 
(3)

$$
\delta_{k}^{\pi}(b, a)=\left\{\begin{array}{cl}
0, & \text { if } \delta_{k}\left(x_{i}, x_{i+1}\right)=0, \text { for every } i=1, \ldots, j-1, \\
\frac{1}{\sum_{i=1}^{j-1} \mathcal{Z}_{k}\left(x_{i}, x_{i+1}\right)} \cdot \sum_{i=1}^{j-1} \delta_{k}\left(x_{i}, x_{i+1}\right), & \text { otherwise. }
\end{array}\right.
$$

According to (2), we obtain

$$
\delta_{k}^{\pi}(b, a)=\left\{\begin{array}{cl}
0, & \text { if } \delta_{k}\left(x_{i}, x_{i+1}\right)=0, \text { for every } i=1, \ldots, j-1, \\
\frac{1}{\sum_{i=1}^{j-1} \mathcal{Z}_{k}\left(x_{i}, x_{i+1}\right)} \cdot \sum_{i=1}^{j-1} \mathcal{Z}_{k}\left(x_{i}, x_{i+1}\right) \cdot \frac{x_{i+1, k}-x_{i k}}{T_{k}-\perp_{k}}, & \text { otherwise. }
\end{array}\right.
$$

Now, starting with $a_{i} \in A$, for some $i=1,2, \ldots, m$, the top element $\top$ can be reached via several different paths with the different running preferences, so we will define the preference of $T$ over $a_{i}$ with respect to the criterion $c_{k}$, $k=1,2, \ldots, n$, as the maximal running preference

$$
\theta_{k}\left(a_{i}, \top\right)=\max _{\pi: a_{i} \rightarrow \top} \delta_{k}^{\pi}\left(a_{i}, \top\right),
$$

and therefore, the preference of $\top$ over the alternative $a$ can be regarded as the $n$-tuple

$$
\theta\left(a_{i}, \top\right)=\left(\theta_{1}\left(a_{i}, \top\right), \theta_{2}\left(a_{i}, \top\right), \ldots, \theta_{n}\left(a_{i}, \top\right)\right) .
$$

The corresponding aggregation function value is given by weighted sum

$$
\alpha\left(a_{i}\right)=\frac{1}{\sum_{k=1}^{n} z_{k}} \cdot \sum_{k=1}^{n} z_{k} \cdot \theta_{k}\left(a_{i}, \top\right) .
$$

On the other hand, for the alternative $a_{i} \in A, i=1,2, \ldots, m$ and the minimal suitable value $P_{q}, q=1,2, \ldots, s$, such that $P_{q} \leq_{A^{n}} a_{i}$, we will extend the definition of running preference in the following way.

By Theorem 2 and Corollary 1, the minimal suitable value $P_{q}$ determines the principal filter $F_{q}$ of $L$ which is generated by $f_{q}$. Let

$$
\pi: f_{q} \longrightarrow a_{i},
$$

be a path from $f_{q}$ to $a_{i}$ via intermediate elements $x_{1}, x_{2}, \ldots, x_{j} \in L$, and let

$$
\pi^{*}: P_{q} \longmapsto f_{q} \longrightarrow a_{i} .
$$

Then the running preference of the alternative $a_{i}$ over the minimal suitable value $P_{q}$ with respect to the criterion $c_{k}, k=1,2, \ldots, n$ trough the path $\pi^{*}$ is defined as 


$$
\delta_{k}^{\pi^{*}}\left(P_{q}, a_{i}\right)=\frac{1}{1+z(\pi)} \cdot \nu_{k}\left(P_{q}, f_{q}\right)+\frac{z(\pi)}{1+z(\pi)} \cdot \delta_{k}^{\pi}\left(f_{q}, a_{i}\right),
$$

where

$$
z(\pi)=\sum_{i=1}^{j-1} \mathcal{Z}_{k}\left(x_{i}, x_{i+1}\right) .
$$

Starting with $P_{q}$, the alternative $a_{i}$ can be reached via several different paths, so we will define the preference of $a_{i}$ over $P_{q}$ with respect to the criterion $c_{k}, k=1,2, \ldots, n$, in the following way

$$
\psi_{k}\left(P_{q}, a_{i}\right)=\min \left\{\min _{\pi^{*}: P_{q} \rightarrow a_{i}} \delta_{k}^{\pi^{*}}\left(P_{q}, a_{i}\right) \mid P_{q} \leq A^{n} a_{i}\right\},
$$

and therefore, the preference of the alternative $a_{i}$ over the minimal suitable value $P_{q}$ can be regarded as the $n$-tuple

$$
\psi\left(P_{q}, a_{i}\right)=\left(\psi_{1}\left(P_{q}, a_{i}\right), \psi_{2}\left(P_{q}, a_{i}\right), \ldots, \psi_{n}\left(P_{q}, a_{i}\right)\right),
$$

and the corresponding aggregation is

$$
\beta\left(a_{i}\right)=\frac{1}{\sum_{k=1}^{n} z_{k}} \cdot \sum_{k=1}^{n} z_{k} \cdot \psi_{k}\left(P_{q}, a_{i}\right) .
$$

Finally, the overall value of alternative $a_{i}$ is determined by

$$
V\left(a_{i}\right)=\beta\left(a_{i}\right)-\alpha\left(a_{i}\right), \quad i=1,2, \ldots, m,
$$

and the rank of alternatives can be obtained by the order relation

$$
a_{i} \prec a_{j} \quad \Longleftrightarrow \quad V\left(a_{i}\right) \leq V\left(a_{j}\right), \quad i, j=1,2, \ldots, m .
$$

\section{Conclusion}

The new method for solving multi-criteria decision making problems introduced in this paper, depend on the minimal suitable values given by the decision maker as an additional piece of information necessary for determining an optimal solution. The method is based on the construction of algebraic lattice generated by the set of the alternative choices. The distance from particular alternative to the ideal solution, and the maximal distance from the alternative to the minimal suitable values, are taken into account in alternative ranking. 


\section{REFERENCES}

[1] J. P. Brans, Ph. Vincke, A preference ranking organization method, Management Science, 1985, 31, 647-656.

[2] W. K. M. Brauers, Optimization methods for a stakeholder society. A revolution in economic thinking by multiobjective optimization: Nonconvex Optimization and its Applications, Kluwer Academic Publishers, Boston, USA, 2004.

[3] D. Dubois, H. Prade, Possibility theory as a basis for qualitative decision theory, Proceedings of the 14th International Joint Conference on Artifcial Intelligence IJCAI'95, Montreal, Canada (1995) 1925-1930.

[4] J. Figueira, S. Greco, M. Ehrgott, Multiple Criteria Decision Analysis: State of the Art Surveys, Springer Verlag, 2005.

[5] M. Grabisch, J.L. Marichal, R. Mesiar, E. Pap, Aggregation Functions, Cambridge University Press, 2009.

[6] I. B. Huang, J. Keisler, I. Linkov, Multicriteria decision analysis in environmental sciences: Ten years of applications and trends, Science of the Total Environment 409 (19) (2011) 3578-3594.

[7] C. L. Hwang, K. Yoon, Multiple Attribute Decision Making Methods and Applications, Springer-Verlag, 1981.

[8] E. P. Klement, R. Mesiar, E. Pap, Measure-based aggregation operators, Fuzzy sets and systems 142 (1) (2004) 3-14.

[9] D. W. Miller, M. K. Starr, Executive Decisions and Operations Research, PrenticeHall, Inc., Englewood Cliffs, NJ, U.S.A., 1969.

[10] G. Munda, Multicriteria evaluation in a fuzzy environment, Heidleberg: PhysicaVerlag, 1995.

[11] S. Opricović, Multicriteria Optimization in Civil Engineering, Faculty of Civil Engineering, Belgrade, 1998 (Monograph in Serbian).

[12] M. Radojičić, M. Žižović, Applications of methods of multi-criteria analysis in bussines decision-making, Technical faculty in Čačak, Serbia, 1998 (Monograph in Serbian).

[13] B. Roy, Multicriteria methodology for decision aiding, Dordecht: Kluwer Academic Publishers, 1996.

[14] T. L. Saaty, The Analytic Hierarchy Process, McGraw-Hill International, New York, NY, U.S.A., 1980.

[15] E. Triantaphyllou, Multi-Criteria Decision Making Methods: A Comparative Study, Kluwer Academic Publishers, Dordrecht, 2000.

[16] N. H. Zardari, K. A. Sharif, M. Shirazi, Z. B. Yusop, Weighting Methods and their Effects on Multi-Criteria Decision Making Model Outcomes in Water Resources Management, Springer, Cham, Heidelberg, New York, Dordrecht, London, 2015. 
[17] M. Zeleny, Multiple Criteria Decision Making, McGrawHill, New York, 1982.

[18] M. Žižović, N. Damljanović, V. Lazarević, N. Deretić, New method for multicriteria analysis, U.P.B. Sci. Bull. Series A 73 (2) (2011) 13-22.

[19] M. M. Žižović, N. Damljanović, M. R. Žižović, Multiplicative multi-criteria analysis method for decision-making, Maejo International Journal of Science and Technology 10 (2) (2016) 233-241.

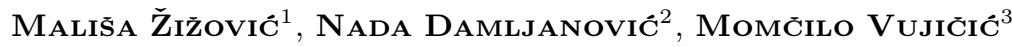
UNIVERSiTy OF KRAGUJEVAC

Faculty of Technical Sciences in ČaČak

SVETOG SAVE 65

32000 С̆AČAK

SERBIA

E-mail address: ${ }^{1}$ zizovic@gmail.rs

${ }^{2}$ nada.damljanovic@ftn.kg.ac.rs

${ }^{3}$ momcilo.vujicic@ftn.kg.ac.rs

Rale Nikolić

Belgrade Metropolitan University

TADEUŠA KošĆUŠKa 63

11000 BELGRADE

SERBia

E-mail address: rale.nikolic@metropolitan.ac.rs 